\title{
On existence and continuation of solutions of the state-dependent impulsive dynamical system with boundary constraints
}

\author{
Ling Chen ${ }^{1}$, Zhi Long He${ }^{1}$, Chuan Dong $\mathrm{Li}^{1 *}$ and Hafiz Gulfam Ahmad Umar ${ }^{2}$
}

"Correspondence: cdli@swu.edu.cn

'National and Local Joint Engineering Laboratory of

Intelligent Transmission and Control Technology (Chongqing), College of Electronic and Information Engineering, Southwest University, Chongqing, P.R. China

Full list of author information is available at the end of the article

\begin{abstract}
The state-dependent impulsive dynamical system with boundary constraints is a kind of special but common system in nature. But because of the complexity of the geometry or topological structures of the impulsive surface, it is hard to determine when an event or an impulsive surface is reached. Therefore, a general state-dependent impulsive nonlinear dynamical system is rarely studied. This paper presents a class of state-dependent impulsive dynamical systems with boundary constraints. We obtain the existence and continuation of their viable solutions and provide sufficient conditions for the existence and uniqueness of the viable solutions to the system. Finally, two examples are given to illustrate the effectiveness of the results.
\end{abstract}

Keywords: Viable solution; State-dependent impulsive; Existence; Continuation; Boundary constraints

\section{Introduction}

The impulsive conditions are not only involved in ordinary differential equations, these conditions may be involved in fractional differential equations as well as in partial differential equations [1, 2]. Impulsive differential equations (IDEs) are basic dynamical models to describe the dynamics of kinds of evolution processes which experience a change of state suddenly, such as harvesting, vibro-impact, natural disasters. These processes are subject to abrupt changes, which are also called perturbations. Since the duration of shortterm perturbations is negligible compared to the duration of an entire evolution [3-5], such perturbations involved in the models are generally expressed in the form of impulses. Impulsive differential equations play a very important role in the model construction and analysis of impulsive problems in electrical, mechanical, population dynamics, industrial robotics, biotechnology, optimal control, pharmacokinetics, economic and social sciences, and so on $[6,7]$, and they have been extensively studied in the past several years [8-12].

Impulsive differential systems have many kinds of different characteristics of impulsive perturbations, and we usually study three kinds of impulsive differential systems: differential systems with fixed-time impulses, differential systems with variable-time impulses, and differential systems with state-dependent impulses. Most of the previous papers [13-

(c) The Author(s) 2019. This article is distributed under the terms of the Creative Commons Attribution 4.0 International License (http://creativecommons.org/licenses/by/4.0/), which permits unrestricted use, distribution, and reproduction in any medium, provided you give appropriate credit to the original author(s) and the source, provide a link to the Creative Commons license, and indicate if changes were made. 
17] consider differential systems with fixed-time impulses or variable-time impulses and discuss their basic qualitative problems, for example, the existence and uniqueness of the solutions of systems, stability, synchronization, bifurcation, etc.

In reality, however, the state-dependent impulsive systems (the impulsive moments depend on the state of the system) are more reasonable in modeling and control due to the state-dependent impulsive control strategy being more economic, efficient, and practical. So far, the state-dependent impulsive systems have a number of applications especially in ecological models, mathematical biology, control theory, etc. In ecological models, the control strategies (by catching, spraying pesticide, or releasing the natural enemy) are taken only when the number of species reaches a critical level, rather than the usual fixed-time impulsive control strategy [18-24]. In particular, Tang et al. [18] studied the existence and stability of positive order- $k(k \geq 1)$ periodic solutions of state-dependent impulsive models by using the properties of the Lambert W function and Poincaré map. Nie et al. [20] studied the existence and stability of positive order-1 or order-2 periodic solution of an SIR epidemic model with state-dependent pulse vaccination. In Chap. 8 of the book Principles of Discontinuous Dynamical Systems, Akhmet [24] studies discontinuous dynamical systems (DDS). The author mainly analyzes the dynamical properties of the solution trajectory and vector field of autonomous equations with discontinuities and studies the local existence, uniqueness, and extension by using the related properties of discontinuous flows (DF). However, due to the complexity of the topological structure of the impulsive hypersurface, the discontinuous dynamical systems this work considers are almost a two-dimensional system, while the study about high dimensional autonomous systems with discontinuous properties is still rare.

Motivated by the above discussions, this paper further studies the viability problem of solutions for general state-dependent impulsive autonomous differential systems with state constraints by combining the relevant research methods in the book Discontinuous Dynamical Systems. For a prescribed open connected subset $\mathcal{K}$ (the viability constraints) of a state space $\mathbb{R}^{n}$, the aim of this paper is to obtain the solutions of differential systems with state-dependent impulses to remain in the viability constraints $\mathcal{K}$ forever. That is to say, when the trajectories of systems do not leave the viability constraints $\mathcal{K}$ or do not reach the boundary $\partial \mathcal{K}$ of $\mathcal{K}$, the solutions of the systems are viable. If the trajectories of systems reach this boundary and leave the viability constraints $\mathcal{K}$ in finite time, the solutions of systems will not be viable (or eventually die out) in the $\mathcal{K}$. We take a reasonable control strategy on the state of systems when the evolution of state $x(t)$ reaches the boundary of $\mathcal{K}$, that is, when $x(t)$ reaches $\mathcal{M} \subset \partial \mathcal{K}$ at time $t_{k}(\mathcal{M}), x(t)$ is reset to $x\left(t^{+}\right)=J\left(x\left(t^{-}\right)\right)$. Under this strategy, we consider state-dependent impulsive autonomous differential systems with state constraints that are governed by the following:

$$
\begin{cases}\dot{x}(t)=f(x), & x(t) \in \mathcal{K}, \text { a.e. } t \geq 0, \\ x\left(t^{+}\right)=J\left(x\left(t^{-}\right)\right), & x\left(t^{-}\right) \in \mathcal{M} \subset \partial \mathcal{K}, t \geq 0, \\ x\left(t_{0}^{+}\right)=x_{0}, & t_{0} \geq 0,\end{cases}
$$

where $f: \mathbb{R}^{n} \rightarrow \mathbb{R}^{n}$ is a sufficiently smooth vector field. For analytical simplification, without loss of generality, we assume that every solution $x(t)$ of (1.1) is right continuous, $x\left(t^{-}\right) \triangleq \lim _{\delta \rightarrow 0} x(t-\delta), x\left(t^{+}\right) \triangleq \lim _{\delta \rightarrow 0} x(t+\delta)=J\left(x\left(t^{-}\right)\right)=x(t), x \in \mathcal{M}$, and $t$ is a moment of discontinuity. $\mathcal{K}$ is regarded as the viability constraints, $\mathcal{M} \subset \partial \mathcal{K}$ is a smooth hypersurface 
in $\mathbb{R}^{n}\left((n-1)\right.$-dimensional submanifold contained in $\left.\mathbb{R}^{n}\right)$ and is called the impulsive surface. $J: \mathcal{M} \rightarrow \mathcal{K}$ is the jump operator for a function $x(t)$. If $x\left(t^{-}\right) \in \mathcal{M}$, then for all $t \geq t_{0}$, we have $x\left(t^{+}\right)=J\left(x\left(t^{-}\right)\right)$. Denote $\mathcal{N} \triangleq J(\mathcal{M}) \in \mathcal{K}$. If $x\left(t^{+}\right) \in \mathcal{N}$, then for any $t \leq t_{0}$, we have $x\left(t^{-}\right)=J^{-1}\left(x\left(t^{+}\right)\right) . x\left(t_{0}^{+}\right)=x_{0} \in \mathcal{K}$ is an initial condition of (1.1). Here we can regard the existence and continuation problem of solutions for state-dependent impulsive autonomous differential system (1.1) with state constraints as a viability problem [25-30].

The paper is structured as follows. Section 2 provides the necessary notations and definitions. In Sect. 3, sufficient conditions for the existence and continuation of viable solution of state-dependent impulsive autonomous differential system (1.1) with state constraints are presented and proved. In Sect. 4, in order to illustrate our results, an example is delivered to illustrate the conclusion.

\section{Preliminaries}

This section introduces some relevant notations, assumptions, and definitions that are necessary for developing the results of this paper. Let $\mathbb{R}$ denote the set of real numbers, let $\mathbb{R}_{+}$be the set of all nonnegative real numbers, let $\mathbb{R}^{n}$ denote the $n$-dimensional phase space, $n \geq 1$. We write $\|\cdot\|$ for the Euclidean vector norm, that is, for a vector $x=\left(x_{1}, x_{2}, \ldots, x_{n}\right) \in \mathbb{R}^{n},\|x\|=\sqrt{x_{1}^{2}+x_{2}^{2}+\cdots+x_{n}^{2}} \cdot \mathcal{B}_{\varepsilon}(\alpha), \alpha \in \mathbb{R}^{n}, \varepsilon>0$, denote the open ball centered at $\alpha$ with radius $\varepsilon .\langle\cdot, \cdot\rangle$ is the standard inner product in $\mathbb{R}^{n}$. For $x \in \mathbb{R}^{n}$ and $\mathcal{K} \subset \mathbb{R}^{n}$, let $d_{\mathcal{K}}(x)$ denote the distance of the point $x$ to the set $\mathcal{K}$ defined by

$$
\mathrm{d}_{\mathcal{K}}(x)=\inf _{y \in \mathcal{K}}\|x-y\|
$$

Firstly, we consider the nonlinear dynamical system

$$
\dot{x}(t)=f(x(t)), \quad x\left(t_{0}\right)=x_{0}, \quad t \in \mathbb{R},
$$

$f: \mathbb{R}^{n} \rightarrow \mathbb{R}^{n}$ is Lipschitz continuous on $\mathbb{R}^{n}$, and $I$ is the maximal interval of existence for the solution $x(t)$ of (2.1). For all $t \in \mathbb{R}$, let $\pi: I \times \mathbb{R}^{n} \rightarrow \mathbb{R}^{n}$ be the flow generated by (2.1), where $\pi$ is a continuous function, $\pi\left(t_{0}, x\right)=x$, and $\pi(s, \pi(t, x))=\pi(t+s, x)$ for all $x \in \mathbb{R}^{n}$, and $t, s \in I$. We define the continuous function $\pi_{x_{0}}: \mathbb{R} \rightarrow \mathbb{R}^{n}$ by $\pi_{x_{0}}(t) \triangleq \pi\left(t, x_{0}\right)$, which is called the nonlinear dynamical system (2.1) with initial condition $x\left(t_{0}\right)=x_{0}$. Note that we use the notation $\pi_{x_{0}}, t \in \mathbb{R}$, and $x(t), t \in I$, interchangeably to denote the solution of (1.1) with initial condition $x\left(t_{0}^{+}\right)=x_{0}$.

The positive orbit of (2.1) through the point $x_{0}$ is given by

$$
\Pi^{+}\left(x_{0}, t\right) \triangleq\left\{\pi\left(t, x_{0}\right) \mid \pi\left(t_{0}, x_{0}\right)=x_{0}, t \geq t_{0}\right\} .
$$

We define

$$
\mathcal{M}^{+}\left(x_{0}\right)=\left(\bigcup_{t>t_{0}} \Pi^{+}\left(x_{0}, t\right)\right) \cap \mathcal{M}
$$

and the exit function (the resetting time) $\tau(x): \mathcal{K} \rightarrow\left(t_{0},+\infty\right]$, where $\tau(x)$ is defined as follows. For a point $(\bar{t}, \bar{x})$ on the trajectory of $(2.1), \tau(\bar{x})=\hat{\tau}>\hat{t}$ means that $\pi_{\bar{x}}(\hat{\tau}) \notin \mathcal{M}$ for $\bar{t}<t<\bar{t}+\hat{\tau}$. This means that $\tau(x)$ is the time of the trajectory of (2.1) from the initialization to the first intersection with the impulsive set (the resetting set) $\mathcal{M}$. 
Remark 2.1 According to the definition of $\tau(\cdot)$, we may know that $\tau(x)>0$ for $x \notin \mathcal{M}$ and $\tau(x)=0$ for $x \in \mathcal{M}$. Furthermore, if $\mathcal{M}^{+}(x)=\emptyset$, then $\tau(x)=\infty$.

The impulsive dynamical system (1.1) is called discontinuous dynamical system (DDS) when $\mathcal{M}^{+}\left(x_{0}\right) \neq \emptyset[24,31,32]$.

Definition 2.1 (Viable solution) A solution $\pi_{x_{0}}(t)$ of (2.1) on $\mathbb{R}_{+}$with initial condition $x(0)=x_{0} \in \mathcal{K}$ is said to be viable in the viability constraints $\mathcal{K} \subset \mathbb{R}^{n}$ on $\mathbb{R}_{+}$if, for every time $t \geq 0, \pi_{x_{0}}(t) \in \mathcal{K}$.

It is apparent that DDS (1.1) is equal to the continuous-time dynamical system (2.1) if $\mathcal{M}^{+}\left(x_{0}\right)=\emptyset$. In this case, for every point $x \in \mathcal{M}$, we consider the Bouligand tangent cone

$$
T_{\mathcal{K}}(x):=\left\{v \in \mathbb{R}^{n} \mid \lim _{h \rightarrow 0^{+}} \frac{1}{h} d_{\mathcal{K}}(x+h v)=0\right\} .
$$

Condition (2.2) means that the vector field $f$ is tangent to $\mathcal{M}$. If $f(x) \in T_{\mathcal{K}}(x)$ holds for all $x \in \mathcal{M}$, then all solutions $\pi_{x}(t)$ of $(2.1)$ are viable in $\mathcal{K}$ (see [28, 29]). If $f(x) \notin T_{\mathcal{K}}(x)$, then the trajectory $\Pi^{+}\left(x_{0}, t\right)$ of (2.1) through $x \in \mathcal{M}$ is, in some sense, transversal to $\mathcal{M}$, hence there exists at least one solution of (2.1) leaving the viability constraints $\mathcal{K}$.

For state-dependent impulsive system (1.1), we make the following hypotheses.

(H1) $\mathcal{M} \neq \emptyset$ and there exists a continuously differentiable function $H: \partial \mathcal{K} \rightarrow \mathbb{R}$ such that the hypersurface $\mathcal{M}$ is defined by

$$
\mathcal{M} \triangleq\{x \in \partial \mathcal{K} \mid H(x)=0 \text { and } \nabla H(x) \neq 0\} .
$$

$(H 2) J: \mathcal{M} \rightarrow \mathcal{N}$ is a continuous differentiable function, and $\operatorname{det}\left[\frac{\partial J(x)}{\partial x}\right] \neq 0$ for $x \in \mathcal{M}$.

Assume that (H1) holds, then it follows from the implicit function theorem [33] that, for every $x \in \mathcal{M}$, there exist a number $j$ and a function $h_{x}\left(x_{1}, \ldots, x_{j-1}, x_{j+1}, \ldots, x_{n}\right)$ such that $\mathcal{M}$ is the graph of the function $x_{j}=h_{x}\left(x_{1}, \ldots, x_{j-1}, x_{j+1}, \ldots, x_{n}\right)$ in the neighborhood of $x$. Assume that (H2) holds, then it follows from the inverse function theorem [33] that there exists a unique function $J^{-1}: \mathcal{N} \rightarrow \mathcal{M}$ such that $\left(J^{-1} \circ J\right)(x)=x$ and $J^{-1}(x) \neq x$ for all $x \in \mathcal{N}$. Furthermore, if $\widetilde{H}(x)=H\left(J^{-1}(x)\right)$ for any $x \in \mathcal{N}$, then $\mathcal{N}=\{x \in \mathcal{K} \mid \widetilde{H}(x)=0\}$. It follows from (2.3) that we can easily prove that $\nabla \widetilde{H}(x) \neq 0[24,31]$. Furthermore, we make the following assumptions:

(H3) $\overline{\mathcal{N}} \cap \mathcal{M}=\emptyset$, where $\overline{\mathcal{N}}$ is the closure of $\mathcal{N}$.

(H4) The vector field $f(x)$ satisfies the following transversality condition: $\langle\nabla H(x), f(x)\rangle \neq$ 0 for all $x \in \mathcal{M}$.

(H5) For $x \in \mathcal{N},\langle\nabla \widetilde{H}(x), f(x)\rangle \neq 0$.

Hypothesis (H3) ensures that when the trajectory of (1.1) meets the surface of $\mathcal{M}$, it instantaneously leaves $\mathcal{M}$. This means that the points of $\mathcal{M}$ are isolated on every trajectory of (1.1). If hypothesis (H4) holds, then $\mathcal{M}$ is said to be transversal to the vector field $f$, it is also called a cross section. In other words, the vector field $f(x)$ is not tangent to the surface $\mathcal{M}$ (see Fig. 1). Hypothesis (H5) implies that the hyperplane $\mathcal{N}$ is not tangent to the solution of (1.1).

In order to define a solution of (1.1), we need the following definition. 
Figure 1 The geometric property of transversal map $f$

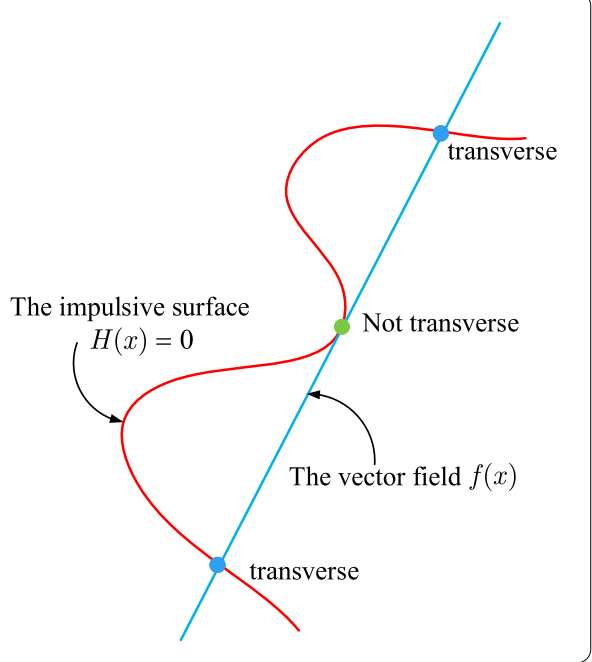

Definition 2.2 A function $\tilde{\pi}_{x_{0}}:\left[t_{0}, t_{f}\right) \rightarrow \mathcal{K}, t_{f} \in \mathbb{R} \cup \infty, t_{f}>t_{0}$, is a solution of (1.1) with initial condition $x\left(t_{0}^{+}\right)=x_{0} \in \mathcal{K}$ if the following conditions are satisfied:

(i) $\tilde{\pi}_{x_{0}}(t)$ is right continuous on $\left[t_{0}, t_{f}\right)$;

(ii) For every $t \in\left[t_{0}, t_{f}\right)$, left and right limits of $\tilde{\pi}_{x_{0}}(t)$ exist, denoted by $\tilde{\pi}_{x_{0}}^{-}(t) \triangleq \lim _{s \rightarrow t^{-}} \tilde{\pi}_{x_{0}}(s)$ and $\tilde{\pi}_{x_{0}}^{+}(t) \triangleq \lim _{s \rightarrow t^{+}} \tilde{\pi}_{x_{0}}(s)$

(iii) There exists a closed discrete subset $\mathcal{I}_{x_{0}} \subset\left[t_{0}, t_{f}\right)$ called impulsive times such that (a) for $t \notin \mathcal{I}_{x_{0}}, \tilde{\pi}_{x_{0}}(t)$ is differentiable, $\frac{\mathrm{d} \tilde{\pi}_{x_{0}}(t)}{\mathrm{d} t}=f\left(\tilde{\pi}_{x_{0}}(t)\right)$, and $\tilde{\pi}_{x_{0}}(t) \notin \mathcal{M}$; (b) for $t \in \mathcal{I}_{x_{0}}, \tilde{\pi}_{x_{0}}^{-}(t) \in \mathcal{M}$ and $\tilde{\pi}_{x_{0}}^{+}(t)=J\left(\tilde{\pi}_{x_{0}}^{-}(t)\right)$.

If $\mathcal{M}^{+}\left(x_{0}\right)=\emptyset$, then $\tilde{\pi}_{x_{0}}(t)=\pi_{x_{0}}(t)$, that is, the trajectory $\widetilde{\Pi}^{+}\left(x_{0}, t\right)$ does not intersect with impulse surface $\mathcal{M}$, there is no impulsive effect. Thus, the trajectory $\widetilde{\Pi}^{+}\left(x_{0}, t\right)$ starting at the initial point $x_{0} \in \mathcal{K}$ will remain in the viability constraints $\mathcal{K}$ forever. Therefore, by the existence and uniqueness theorem for ordinary differential equation, $\tilde{\pi}_{x_{0}}(t)$ exists and is unique on an interval $\left[0, t_{f}\right)$ as a viable solution of system (2.1).

However, if $\mathcal{M}^{+}\left(x_{0}\right) \neq \emptyset$, then $\tau\left(x_{0}\right)<+\infty$. Thus, there exists a smallest positive time $\tau_{1} \triangleq \tau\left(x_{0}\right)$ such that $x_{1} \triangleq \pi_{x_{0}}\left(\tau_{1}\right) \in \mathcal{M}$ and $\pi_{x_{0}}(t) \notin \mathcal{M}$ for $t_{0}<t<\tau_{1}$. Furthermore, $x_{1}$ is instantaneously transferred to $x_{1}^{+} \triangleq J\left(x_{1}\right)$. Then we define $\tilde{\pi}_{x}$ on $\left[t_{0}, t_{1}\right]$ by

$$
\tilde{\pi}_{x_{0}}(t)= \begin{cases}\pi_{x_{0}}(t), & t_{0} \leq t<t_{1}, \\ x_{1}^{+}, & t=t_{1}\end{cases}
$$

where $\tilde{\pi}_{x_{0}}\left(0^{+}\right)=x_{0}$ and $t_{1} \triangleq \tau_{1}$. Further, if $\mathcal{M}^{+}\left(x_{1}^{+}\right)=\emptyset$, then we define $\tilde{\pi}_{x_{0}}(t)=\pi_{x_{1}^{+}}\left(t-\tau_{1}\right)$ for $\tau_{1} \leq t<+\infty$ and $\tau\left(x_{1}^{+}\right)=+\infty$. That is to say, the trajectory $\widetilde{\Pi}^{+}\left(x_{0}, t\right)$ starting at the initial point $x_{0} \in \mathcal{K}$ meets the surface $\mathcal{M}$ only once and does not hit the surface $\mathcal{M}$ beyond the time $t=\tau_{1}$. On the other hand, if $\mathcal{M}^{+}\left(x_{1}^{+}\right) \neq \emptyset$, then there exists a smallest positive time $\tau_{2} \triangleq \tau\left(x_{1}^{+}\right)$such that $x_{2} \triangleq \pi_{x_{1}^{+}}\left(\tau_{2}\right) \in \mathcal{M}$ and $\pi_{x_{1}^{+}}\left(t-\tau_{1}\right) \notin \mathcal{M}$, for $\tau_{1}<t<\tau_{1}+\tau_{2}$. Moreover, $x_{2}$ jumps to point $x_{2}^{+} \triangleq J\left(x_{2}\right)$. Therefore, we define $\tilde{\pi}_{x}$ on $\left[t_{1}, t_{2}\right]$ by

$$
\tilde{\pi}_{x_{0}}(t)= \begin{cases}\pi_{x_{1}^{+}}\left(t-t_{1}\right), & t_{1} \leq t<t_{2}, \\ x_{2}^{+}, & t=t_{2},\end{cases}
$$




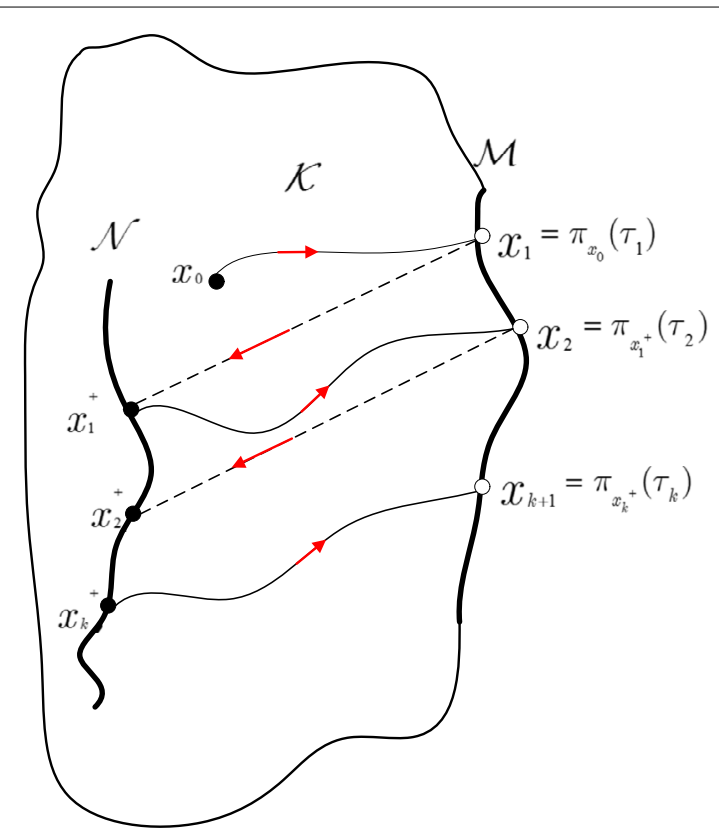

Figure 2 The trajectory $\tilde{\pi}_{x_{0}}(t)$ of (1.1) with the initial condition $x_{0} \in \mathcal{K}$

where $t_{2}=\tau_{1}+\tau_{2}$. Repeating this process for $x_{k}^{+}, k=2,3, \ldots$, we can define $\tilde{\pi}_{x_{0}}$ on each $\left[t_{k}, t_{k+1}\right]$ by the following:

$$
\tilde{\pi}_{x_{0}}(t)= \begin{cases}\pi_{x_{k}^{+}}\left(t-t_{k}\right), & t_{k} \leq t<t_{k+1}, \\ x_{k+1}^{+}, & t=t_{k+1},\end{cases}
$$

where $t_{k}=\sum_{i=1}^{k} \tau_{i}, \tau_{i} \triangleq \tau\left(x_{i-1}^{+}\right), t_{0}=0$, and $x_{k}^{+} \triangleq J\left(x_{k}\right)$. Therefore, the solution $\tilde{\pi}_{x_{0}}(t)$ of (1.1) is defined on the interval $\left[t_{0}, t_{k+1}\right]$ (see Fig. 2). If $\mathcal{M}^{+}\left(x_{k}^{+}\right)=\emptyset$ for some $k$, then the trajectory $\widetilde{\Pi}^{+}\left(x_{0}, t\right)$ of (1.1) with initial condition $x\left(t_{0}^{+}\right)=x_{0} \in \mathcal{K}$ will intersect the impulsive set $\mathcal{M}$ finitely many times ( $k$ times) and will remain in the viability constraints $\mathcal{K}$ forever. Then there exists a solution of (1.1), and $\tilde{\pi}:\left[\tau_{k}, t_{f}\right) \rightarrow \mathcal{K}$ is a maximal solution of (2.1). If $\mathcal{M}^{+}\left(x_{k}^{+}\right) \neq \emptyset$ for all $k=1,2, \ldots$, then $\tilde{\pi}_{x_{0}}(t)$ is defined on the interval $\left[t_{0}, t_{f}\right)$. Furthermore, a maximal interval of the existence of a solution does not exist since $\left[t_{0}, t_{f}\right)$ involves a sequence $\left\{t_{k}\right\}_{k=1}^{\infty}$ of impulsive times, where $t_{k}=\sum_{i=1}^{\infty} \tau_{i}$ and $\lim _{k \rightarrow \infty}=\infty$.

Remark 2.2 Note that $\tilde{\pi}_{x_{0}}\left(t_{k}\right) \in \mathcal{M}, \tilde{\pi}_{x_{0}}\left(t_{k}^{+}\right) \in \mathcal{N}$. Moreover, $\tilde{\pi}_{x_{0}}\left(t_{k}\right) \in \mathcal{M}$ for $t_{k}>t_{0}$ and $\tilde{\pi}_{x_{0}}\left(t_{k}\right) \in \mathcal{N}$ for $t_{k}>t_{0}$, where $t_{k} \in \mathcal{I}_{x_{0}}$.

For given $x_{0} \in \mathcal{K}$, the positive orbit of (1.1) with initial condition $x\left(0^{+}\right)=x_{0} \in \mathcal{K}$ is defined by

$$
\widetilde{\Pi}^{+}\left(x_{0}, t\right)=\left\{\tilde{\pi}_{x_{0}}(t) \mid t \in\left[t_{0}, t_{f}\right)\right\}
$$

We let $t_{k}$ denote the $k$ th instant of time at which $\widetilde{\Pi}^{+}\left(x_{0}, t\right)$ intersects $\mathcal{M}, \mathcal{I}_{x_{0}}$ is denoted by $\left\{t_{1}, t_{2}, \ldots, t_{k}, \ldots\right\}$, where $t_{0}<t_{1}<t_{2}<\cdots<t_{k}<\cdots$ and $\lim _{k \rightarrow \infty} t_{k}=\infty$. 
Remark 2.3 If $t_{f}<\infty$, then $\tilde{\pi}_{x_{0}}:\left[t_{0}, t_{f}\right) \rightarrow \mathbb{R}^{n}$ is a maximal solution of (1.1), where $\mathcal{I}_{x_{0}} \neq \emptyset$, $\tilde{\pi}_{x_{0}}:\left[\max \left(\mathcal{I}_{x_{0}}\right), t_{f}\right) \rightarrow \mathbb{R}^{n}$ is a maximal solution of $(1.1)$, and when $\mathcal{I}=\emptyset, \tilde{\pi}_{x_{0}}:\left[t_{0}, t_{f}\right) \rightarrow \mathbb{R}^{n}$ is a maximal solution of (2.1). If $t_{f}=\infty$, then the solution is obviously maximal.

We shall use $\mathcal{P C}\left(\left[t_{0}, t_{f}\right), \mathbb{R}^{n}\right)$ to denote the class of piecewise continuous functions from $\left[t_{0}, t_{f}\right)$ to $\mathbb{R}^{n}$, with discontinuities of the first kind only at $t=t_{k}, k=1,2, \ldots$ Thus, $\widetilde{\pi}_{x_{0}}(t) \in$ $\mathcal{P C}^{1}\left(\left[t_{0}, t_{f}\right), \mathbb{R}^{n}\right)$.

Now we give the Schauder fixed point theorem, the definitions of the impulsive viable solution and continuation of the solution of (1.1).

Theorem 2.1 (Schauder fixed point theorem [33]) Let $\mathcal{C} \subseteq \mathbb{R}^{n}$ be a nonempty, convex, and closed set, let $f: \mathcal{C} \rightarrow \mathcal{C}$ be continuous, and assume that $f(\mathcal{C})$ is bounded. Show that there exists $x \in \mathcal{C}$ such that $f(x)=x$.

Definition 2.3 (Impulsive viable solution) A solution $\tilde{\pi}_{x_{0}}(t) \in \mathcal{P C} \mathcal{C}^{1}\left(\left[t_{0}, t_{f}\right), \mathbb{R}^{n}\right)$ of $(1.1)$ on the interval $\left[t_{0}, t_{f}\right)$ with initial condition $x\left(0^{+}\right)=x_{0}$ is said to be viable in the viability constraints $\mathcal{K} \subset \mathbb{R}^{n}$ on $\left[t_{0}, t_{f}\right)$ if, for every time $t \in\left[t_{0}, t_{f}\right) \backslash \mathcal{I}_{x_{0}}, \tilde{\pi}_{x_{0}}(t) \in \mathcal{K}$.

Definition 2.4 ([24]) A solution $\tilde{\pi}_{x_{0}}(t)$ of (1.1) is said to be continuable to a set $U \in \mathbb{R}^{n}$ as time decreases (increases) if there exists a time $s \in \mathbb{R}$ such that $s \leq 0(s \geq 0)$ and $\tilde{\pi}_{x_{0}}(s) \in U$.

In order to obtain the sufficient conditions of continuation of the solutions of (1.1), we make the following hypotheses:

(H6) $\sup \|f(x)\|<+\infty$ for all $x \in \mathcal{K}$.

(H7) (a) Every solution $\pi_{x_{0}}(t), x_{0} \in \mathcal{K}$, of (2.1) is continuable to either $\infty$ or $\mathcal{M}$ as time increases.

(b) Every solution $\pi_{x_{0}}(t), x_{0} \in \mathcal{K}$, of (2.1) is continuable to either $-\infty$ or $\mathcal{N}$ as time decreases.

\section{Main results}

In this section we prove the existence and continuation of solution of (1.1).

The following theorem gives sufficient conditions for the existence and uniqueness of solutions of (1.1).

Theorem 3.1 If hypotheses (H1)-(H3) hold, then for every $x_{0} \in \mathcal{K}$, there exist $r<t_{0}$ and $s>t_{0}$ such that (1.1) has a unique viable solution $x:[r, s] \rightarrow \mathcal{K}$ over the interval $[r, s]$.

Proof According to the different position of the initial point $x_{0}, \mathcal{M}$ and $\mathcal{N}$, we consider the following three cases:

(C1) If $x_{0} \notin M \cup N$, then this implies that there exists a constant $\alpha>0$ small enough such that $\mathcal{B}_{\alpha}\left(x_{0}\right) \cap(\mathcal{M} \cup \mathcal{N})=\emptyset$ and $\mathcal{B}_{\alpha}\left(x_{0}\right) \subseteq \mathcal{K}$. Let $M \triangleq \sup \left\{\|f(x)\|: x \in \mathcal{B}_{\alpha}\left(x_{0}\right)\right\}$. Further, let $\xi, \eta>0$ be such that $M \xi \leq \eta \leq \varepsilon$, and let

$$
\Omega \triangleq\left\{x(\cdot) \in C\left[t_{0}, s\right] \mid\left\|x-x_{0}\right\| \leq \alpha, x\left(t_{0}\right)=x_{0}, t \in\left[t_{0}, s\right]\right\}
$$


where $s \triangleq t_{0}+\xi$. It is easy to see that $\Omega$ is a convex closed set and bounded. Let $G: C\left[t_{0}, s\right] \rightarrow C\left[t_{0}, s\right]$ be given by

$$
(G x)(t) \triangleq x_{0}+\int_{t_{0}}^{t} f(x(v)) \mathrm{d} v, \quad t \in\left[t_{0}, s\right]
$$

It follows that

$$
\begin{aligned}
\left\|(G x)(t)-x_{0}\right\| & =\left\|\int_{t_{0}}^{t} f(x(v)) \mathrm{d} v\right\| \\
& \leq \int_{t_{0}}^{t}\|f(x(v))\| \mathrm{d} v \\
& \leq M\left|t-t_{0}\right| \\
& \leq M \xi \\
& \leq \eta,
\end{aligned}
$$

where $t \in\left[t_{0}, s\right], G(\Omega)$ is bounded by (3.2). Furthermore, because $f$ is continuous on $\mathcal{K}$, it follows that, for every $\varepsilon>0$, there exists $\delta>0$ such that $\sup _{t_{0} \leq t \leq s}\|x(t)-\bar{x}(t)\|<\delta$, we have

$$
\begin{aligned}
\|(G x)(t)-(G \bar{x})(t)\| & =\| \int_{t_{0}}^{t}[f(x(v))-f(\bar{x}(v)) \mathrm{d} v \| \\
& \leq \int_{t_{0}}^{t}\|f(x(v))-f(\bar{x}(v))\| \mathrm{d} v \\
& \leq \varepsilon\left(t-t_{0}\right) \\
& \leq \varepsilon \xi .
\end{aligned}
$$

Therefore, by the Schauder fixed point theorem 2.1, we know that $x(t)=(G x)(t)$ is a solution of (1.1) if and only if $x(t)$ is a fixed point of $G$ for all $t \in\left[t_{0}, s\right]$ (for more details, see [33]). On the other hand, according to the uniqueness theorem of nonlinear dynamical system (2.1), we obtain that system (1.1) has a unique solution $x:\left[t_{0}, s\right] \rightarrow \mathbb{R}^{n}$ over the interval $\left[t_{0}, s\right]$.

(C2) If $x_{0} \in \mathcal{M}$, then for all $t \geq t_{0}$, we have $x_{0}^{+}=J\left(x_{0}\right) \in \mathcal{N} \in \mathcal{K}$. It follows from hypothesis (H3) that there exists a constant $\alpha>0$ such that $\mathcal{B}_{\alpha}\left(x_{0}^{+}\right) \cap \mathcal{M} \neq \emptyset$ and $\mathcal{B}_{\alpha}\left(x_{0}^{+}\right) \subseteq \mathcal{K}$. Hence, $x(t)$ can be continued at the right. Similar to (C1), (1.1) has a unique solution $x:\left[t_{0}, s\right] \rightarrow \mathbb{R}^{n}$ over the interval $\left[t_{0}, s\right]$. Let us consider $t \leq t_{0}$ now. It is easy to see that $x_{0}^{-}=J^{-1}\left(x_{0}^{+}\right) \in \mathcal{M}$. Hence, there exists a constant $\alpha>0$ such that $B_{\alpha}\left(x_{0}^{+}\right) \cap \mathcal{N} \neq \emptyset$ by hypothesis $(H 3)$, and $x(t)$ can be proceeded at the left. This means that there exists a constant $\xi$ such that $r \triangleq t_{0}-\xi$. Similar to (C1), (1.1) has a unique solution $x:\left[r, t_{0}\right] \rightarrow \mathbb{R}^{n}$ over the interval $\left[r, t_{0}\right]$. Therefore, there exists a unique solution $x(t)$ of (1.1) on an interval $(r, s)$.

(C3) If $x_{0} \in \mathcal{N}$, similar to cases (C1) or (C2).

Through the analyses above, we proved that (1.1) has a unique solution $x:[r, s] \rightarrow \mathbb{R}^{n}$ over the interval $[r, s]$. The proof is complete. 
Remark 3.1 If hypotheses (H1)-(H5) hold, then every solution of (1.1) continuously depends on the initial value $x_{0}[24,31]$.

Next, we discuss the continuation of the solution for (1.1). The following theorems prove that every solution of (1.1) is a continuation to $\mathbb{R}$.

Theorem 3.2 If hypotheses (H4), (H6), and (H7) hold, then every solution $\pi_{x_{0}}(t), x_{0} \in \mathcal{K}$ of (2.1) is continuable to $\mathbb{R}$.

Proof Let $\tilde{\pi}_{x_{0}}(t)$ be a solution of system (2.1) starting from the initial point $x_{0} \in \mathcal{K}$ at $t=0$. Since the solution $\pi_{x_{0}}(t)$ intersects the impulse surface $\mathcal{M}$ zero times or finitely many times, or infinitely many times, thus, the relation of $\tilde{\pi}_{x_{0}}(t)$ and $\mathcal{M}$ is one of the following three cases:

(i) If $\tilde{\pi}_{x_{0}}(t)$ does not intersect the impulse set $\mathcal{M}$, then the solution $\tilde{\pi}_{x_{0}}(t)$ of system (2.1) starting at the initial point $x_{0} \in \mathcal{K}$ is free from the impulsive effects and remains in the set $\mathcal{K}$ forever. It means that $\tilde{\pi}_{x_{0}}(t)$ is a nonlinear dynamical system (2.1). According to hypotheses (H7), the solutions of (1.1) on the maximal interval $\left[t_{0}, t_{f}\right)$ of existence are continuable to $\mathbb{R}$.

(ii) If the solution of (2.1) intersects the impulse surface $\mathcal{M}$ at the time $t_{k}$ (i.e., $\left.\tilde{\pi}_{x_{0}}\left(t_{k}\right) \in \mathcal{M}\right)$ only finitely many times, where the impulse time sequence $\left\{t_{k}\right\} \in \mathbb{R}$ satisfies $-\infty<t_{1}<t_{2}<\cdots<t_{k}<+\infty$. Denote by $t_{\min }$ and $t_{\max }$ the minimal and maximal elements of the sequence $\tau_{i}$, respectively. For $t \geq t_{\max }$, the solution $\tilde{\pi}_{x_{0}}\left(t_{\max }\right)$ of system (2.1) is subjected by impulsive effect to jump to $x\left(t_{\max }^{+}\right)=J\left(x\left(t_{\text {max }}^{-}\right)\right) \in \mathcal{N}$, and the solution $\tilde{\pi}_{x_{0}}(t)=\pi_{x\left(t_{\max }^{+}\right)}(t)$ of system (2.1), where $\pi_{x\left(t_{\max }^{+}\right)}(t)$ is a solution of $(2.1)$. By hypothesis $(H 7)(a),\left[t_{0}, t_{f}\right)$ is continuable to $\left[t_{0}, \infty\right)$ for $t \leq t_{\min }$. Similarly, by hypothesis $(H 7)(b),\left[t_{0}, t_{f}\right)$ is also continuable to $\left(-\infty, t_{0}\right]$.

(iii) The solution $\tilde{\pi}_{x_{0}}(t)$ of system (1.1) intersects the impulse surface $\mathcal{M}$ infinitely many times. It is clear that the existence of $\tau_{\min }$ and $\tau_{\max }$ has the following three cases:

(a) The impulse time sequence $\left\{t_{k}\right\}$ has a maximal element $t_{\max } \in \mathbb{R}$, but $t_{\min }$ does not exist. According to the proof of case (ii), we know $\tilde{\pi}_{x_{0}}(t)$ is continuable to $+\infty$ as $t$ increases. Consider $t$ to be decreasing. Integrating both sides of the ordinary differential equation of system (2.1) that belongs to the interval $\left[t_{k}, t_{k+1}\right)$, we have

$$
\tilde{\pi}_{x_{0}}\left(t_{k}^{+}\right)=\tilde{\pi}_{x_{0}}\left(t_{k+1}^{-}\right)+\int_{t_{k+1}}^{t_{k}} f\left(\tilde{\pi}_{x_{0}}(\theta)\right) \mathrm{d} \theta .
$$

From $(H 4)$ and $(H 6)$, we denote $Q \triangleq \sup _{\mathcal{K}}\|f(x)\|$ and $\rho \triangleq \mathrm{d}(\mathcal{M}, \mathcal{N})>0$. Thus, (3.3) implies that

$$
\frac{\rho}{Q} \leq\left(t_{k+1}-t_{k}\right)
$$

Therefore,

$$
\frac{\rho}{Q}\left(k-k^{*}\right) \geq t_{k}-t_{k^{*}},
$$


where $k^{*}$ is fixed, $k<k^{*}$, and $k, k^{*}$ is the index of the impulse time sequence $\left\{t_{k}\right\}$ and is fixed. From (3.4), this implies that $t_{k} \rightarrow-\infty$ as $k \rightarrow-\infty$. According to hypothesis $(H 7)(b),\left[t_{0}, t_{f}\right)$ is continuable to $\left(-\infty, t_{\max }\right)$. Thus, $\tilde{\pi}_{x_{0}}(t)$ is continuable to $-\infty$ as $t$ decreases.

(b) The sequence $\left\{t_{k}\right\}$ has a minimal element $t_{\min } \in \mathbb{R}$, but does not have a maximal one. Then by the arguments of (ii) $x(t)$ is continuable to $-\infty$. It follows now that we consider the continuation of $x(t)$ with the increasing of time $t$. We have

$$
\tilde{\pi}_{x_{0}}\left(t_{k+1}^{-}\right)=\tilde{\pi}_{x_{0}}\left(t_{k}^{+}\right)+\int_{t_{i}}^{t_{k+1}} f\left(\tilde{\pi}_{x_{0}}(\theta)\right) \mathrm{d} \theta
$$

Similarly, we have

$$
\frac{\rho}{Q} \leq t_{k+1}-\tau_{k}
$$

or

$$
\frac{\rho}{Q}\left(k-k^{*}\right) \leq t_{k}-t_{k^{*}}
$$

where $k^{*}$ is fixed, and $k>k^{*}$. From (3.5), we get $t_{k} \rightarrow+\infty$ as $k \rightarrow+\infty$.

According to hypothesis $(H 7)(a),\left[t_{0}, t_{f}\right)$ is continuable to $\left[t_{\min }, \infty\right)$.

(c) The sequence $\left\{t_{k}\right\}$ has neither a minimal nor a maximal element. The proof of this case is similar to that of (a) and (b). We obtain that $\tilde{\pi}_{x_{0}}(t)$ is continuable to $\mathbb{R}$.

According to the above discussion, we obtain that every solution $\tilde{\pi}_{x_{0}}(t)$ of $(1.1)$ is continuable to $\mathbb{R}$. This completes the proof.

The main results claim that every viable solution of (1.1) is continuable to $+\infty$ and $-\infty$. In other words, $\mathbb{R}$ is a maximal interval of existence of each solution $\tilde{\pi}_{x_{0}}(t), x_{0} \in \mathcal{K}$ of (1.1). That is, $\tilde{\pi}_{x_{0}}(t) \in \mathcal{P C}(\mathbb{R})$.

\section{Numerical examples}

In this section, the validity of the results will be illustrated by two numerical examples.

Example 4.1 We revisit the following state-dependent impulsive autonomous differential system with state constraints [31]:

$$
\left\{\begin{array}{l}
\dot{x}=-x-3 y \\
\dot{y}=3 x-y \\
x^{+}=2 x \\
y^{+}=2 y
\end{array}\right\}(x, y) \in \mathcal{K},
$$

where $z=(x, y)$,

$$
\mathcal{K}=\left\{(x, y) \in \mathbb{R}^{2} \mid 1<x^{2}+y^{2} \leq 4\right\} \quad \text { and } \quad \mathcal{M}=\left\{(x, y) \in \mathbb{R}^{2} \mid x^{2}+y^{2}=1\right\}
$$


It is easy to find that

$$
\begin{aligned}
& f(z)=(-x-3 y, 3 x-y), \quad J(x)=(2 x, 2 y), \quad \mathcal{N}=\left\{(x, y) \in \mathbb{R}^{2} \mid x^{2}+y^{2}=4\right\}, \\
& H(x)=x^{2}+y^{2}-1, \quad \tilde{H}(x)=x^{2}+y^{2}-4 .
\end{aligned}
$$

The viability constraints of system $(4.1)$ are $\mathcal{K}=\left\{(x, y) \in \mathbb{R}^{2} \mid 1<x^{2}+y^{2} \leq 4\right\}$, manifolds $\mathcal{M}$ and $\mathcal{N}$ are boundaries of the set $\mathcal{K}$, and circles with radii 1 and 2 , respectively. It is easy to $\operatorname{know} \mathrm{d}(\mathcal{M}, \mathcal{N})=1>0$.

Let us check conditions $(H 1)-(H 6)$. Clearly, $\mathcal{K}=\left\{(x, y) \in \mathbb{R}^{2} \mid 1<x^{2}+y^{2} \leq 4\right\}$ is a simply connected open subset on $\mathbb{R}^{n}$. Moreover,

$$
\nabla H(x)=(2 x, 2 y) \neq 0 .
$$

So, $(H 1)-(H 2)$ are satisfied. Moreover, $f, J$ are continuously differentiable functions and

$$
\operatorname{det}\left[\frac{\partial J(x)}{\partial x}\right]=\left|\begin{array}{ll}
2 & 0 \\
0 & 2
\end{array}\right| \neq 0
$$

for all $x$. Furthermore, for all $x \in \mathcal{M}$, we have

$$
\langle\nabla H(x), f(x)\rangle=\langle(2 x, 2 y),(-x-3 y, 3 x-y)\rangle=2\left(-x^{2}-y^{2}\right)=-2 \neq 0,
$$

and

$$
\langle\nabla \tilde{H}(x), f(x)\rangle=\langle(2 x, 2 y),(-x-3 y, 3 x-y)\rangle=2\left(-x^{2}-y^{2}\right)=-8 \neq 0
$$

for all $x \in \mathcal{N}$. Thus, all conditions (H1)-(H6) are satisfied. It is easy to know from Theorem 3.1 that solution of system (4.1) exists and is unique.

Next, let us consider the continuation of solution of system (4.1). The nonlinear dynamical system in (4.1) is a linear dynamical system with constant coefficients. Furthermore,

$$
\|f(x)\|=\sqrt{(-x-3 y)^{2}+(3 x-y)^{2}}=\sqrt{10\left(x^{2}+y^{2}\right)}
$$

and

$$
\sup \|f(x)\|=2 \sqrt{10}<\infty, \quad x \in \mathcal{K} .
$$

We see that system (4.1) satisfies all the conditions of Theorem 3.2. Therefore, every solution of system (4.1) is continuable on $\mathbb{R}$.

The phase portrait of system (4.1) without impulsive effects is seen in Fig. 3(a). Figure 3(a) shows that the trajectories of system (4.1) without impulsive effect will leave $\mathcal{K}$ and then trend to equilibrium $(0,0)$. From Fig. 3(b), we can easily find that the trajectory of system (4.1) starting from the initial point $(\sqrt{2}, \sqrt{2}) \in \mathcal{K}$ will intersect with the curve $x^{2}+y^{2}=1$ infinitely many times due to impulsive effects and remain in $\mathcal{K}$ forever. That is to say, we can make the solution of system (4.1) with boundary constraints remain in the constraint domain $\mathcal{K}$ through the strategy of state-dependent impulsive control. 


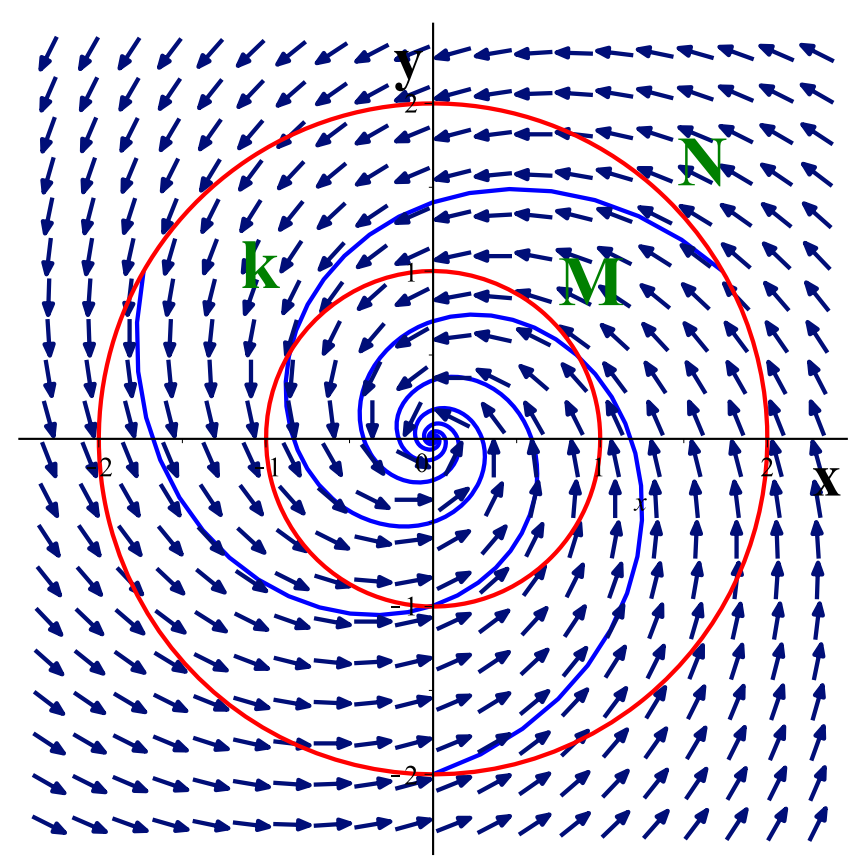

(a) The vector fields of the system (4.1) without impulsive effects

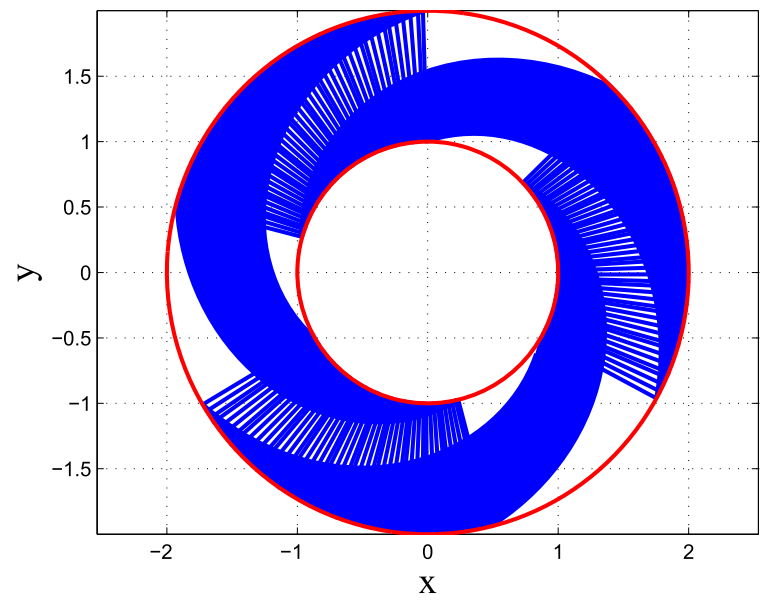

(b) The trajectory of the system (4.1) with initial conditions $\left(x_{0}, y_{0}\right)=(\sqrt{2}, \sqrt{2})$

Figure 3 The numerical simulation of system (4.1)

To further illustrate the significance of the study, we consider a specific biological model.

Example 4.2 Suppose that there are two species in an area with limited food resources. $x_{1}(t)$ and $x_{2}(t)$ are the populations of the species at time $t$, respectively. Let $x_{1}=x_{1}(t)$ and $x_{2}=x_{2}(t)$. Suppose that the species $x_{2}$ has the negative effect on the species $x_{1}$ and decreases the growth rate of the species $x_{1}$, but the species $x_{1}$ can increase the growth rate of the species $x_{2}$. The relations of two species can be described by the following system:

$$
\left\{\begin{array}{l}
\dot{x}_{1}=-\left(x_{2}-a_{2}\right)+\epsilon\left(x_{1}-a_{1}\right), \\
\dot{x}_{2}=x_{1}-a_{1},
\end{array}\right.
$$




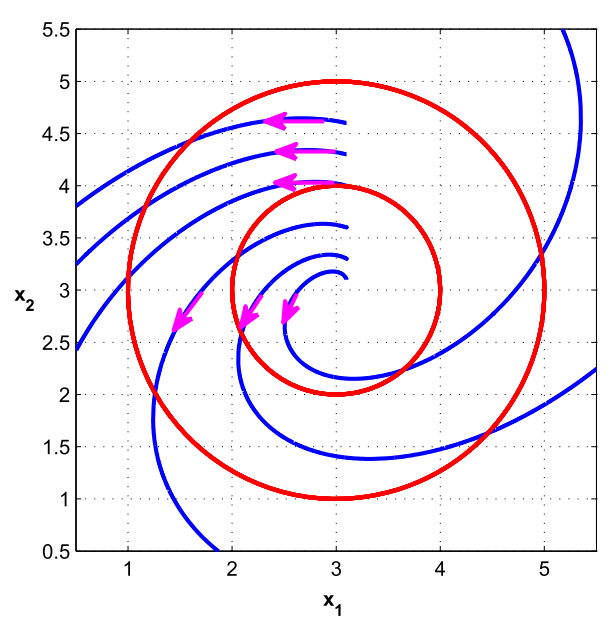

(a) The trajectories of the system (4.4) without impulsive effects

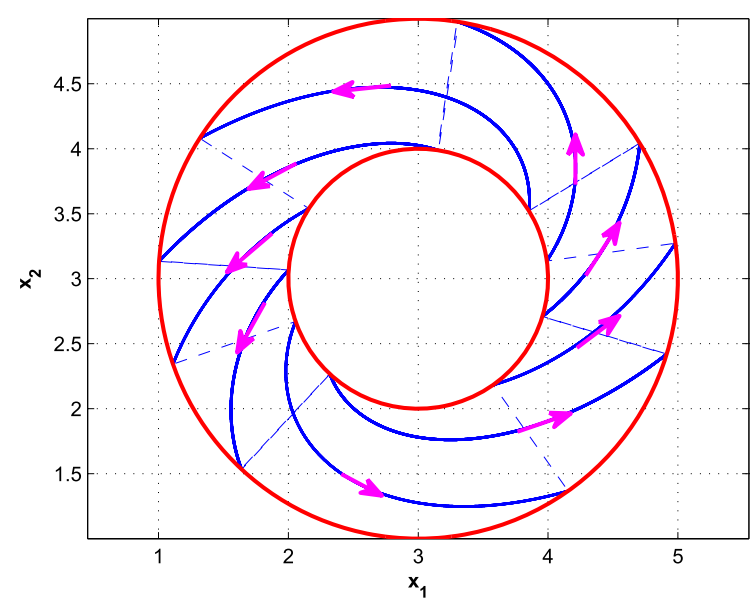

(b) The trajectory of the system (4.4) with state-dependent impulsive control

Figure 4 The numerical simulation of system (4.4), where $a_{1}=a_{2}=3, \epsilon=0.7, \rho_{1}=2, \rho_{2}=1$

where $\epsilon>0$ represents the immigration rate of species $x_{1}$ from the outside of the habitat, $a_{1}, a_{2}$ are given positive constants. It is easy to know that the equilibrium point $\left(a_{1}, a_{2}\right)$ of system (4.3) is unstable focus or node for $\epsilon>0$ (see Fig. 4(a)). That is to say, $x_{1} \rightarrow+\infty$ and $x_{2} \rightarrow+\infty$ as $t \rightarrow \infty$. However, the number of the two species will decrease or even tend to extinction after the total population reaches a certain threshold because the food is limited. In order to ensure the diversity of species, we should take some reasonable control strategies. We assume that the threshold $x_{1}^{2}+x_{2}^{2}=\rho_{1}^{2}, \rho_{1}>0$. When $x_{1}$ and $x_{2}$ satisfy the threshold, some control strategies can be taken, and the population of two species decreases to $x_{1}^{2}+x_{2}^{2}=\rho_{2}^{2}$, where $\rho_{1}>\rho_{2}>0$ ( $\rho_{1}$ and $\rho_{1}$ are constants). Therefore, we give the following cylindrical dynamical system with state-dependent impulsive control and state constraints:

$$
\left\{\begin{array}{l}
\dot{x}_{1}=-\left(x_{2}-a_{2}\right)+\epsilon\left(x_{1}-a_{1}\right) \\
\dot{x}_{2}=x_{1}-a_{1}, \\
\Delta \rho=-\rho^{*}, \quad(x, y) \in M \subset \partial \mathcal{K},
\end{array}\right\}(x, y) \in \mathcal{K},
$$


where

$$
\begin{aligned}
& \mathcal{K}=\left\{\left(x_{1}, x_{2}\right) \in \mathbb{R}_{+}^{2} \mid\left(x_{1}-a_{1}\right)^{2}+\left(x_{2}-a_{2}\right)^{2}<\rho_{1}^{2}\right\}, \\
& \Delta \rho=-\rho^{*}=\rho_{2}-\rho_{1}=\sqrt{\left(x_{1}^{+}-a_{1}\right)^{2}+\left(x_{2}^{+}+a_{2}\right)^{2}}-\sqrt{\left(x_{1}-a_{1}\right)^{2}+\left(x_{2}-a_{2}\right)^{2}}, \\
& \partial \mathcal{K}=\left\{\left(x_{1}, x_{2}\right) \in \mathbb{R}_{+}^{2} \mid\left(x_{1}-a_{1}\right)^{2}+\left(x_{2}-a_{2}\right)^{2}=\rho_{1}^{2}\right\},
\end{aligned}
$$

and

$$
\mathcal{M}=\left\{\left(x_{1}, x_{2}\right) \in \mathbb{R}_{+}^{2} \mid\left(x_{1}-a_{1}\right)^{2}+\left(x_{2}-a_{2}\right)^{2}=\rho_{1}^{2}\right\} .
$$

It is easy to see that assumptions (H1)-(H6) are true. Let $a_{1}=a_{2}=3, \epsilon=0.7, \rho_{1}=2$, $\rho_{2}=1$. Figure 4(a) shows that the solution of system (4.4) starting from the initial value $\left(x_{10}, x_{20}\right) \in \mathcal{K}$ will leave the viability constraints $\mathcal{K}$. Figure 4 (b) shows that the solution of system (4.4) will eventually stay in the viability constraints $\mathcal{K}$ and tend to a periodic solution.

\section{Conclusion}

The state-dependent impulsive autonomous differential system (1.1) with boundary constraints has been considered in this paper. The main purpose is to investigate the existence and uniqueness of viable solutions of system (1.1). From Theorem 3.1, some sufficient conditions on the existence of viable solutions of system (1.1) are provided. Furthermore, we obtain sufficient conditions for the continuation of a viable solution of system (1.1) by Theorem 3.2. Finally, two examples are given to illustrate the existence and continuation of viable solutions of (1.1).

\section{Acknowledgements}

The authors are grateful to the anonymous referees for their careful reading of the manuscript and for their invaluable comments and suggestions, which largely helped to improve this paper.

\section{Funding}

This work is supported by the Natural Science Foundation of China (grant no: 61873213, 61503307, 61633011, 61702066, 11474233, 11747125), Chongqing Research Program of Basic Research and Frontier Technological Science Foundation (cstc2016jcyjA0261, cstc2017jcyjAX0256). It is also supported by Fundamental Research Funds for the Central Universities (no: XDJK2019B009) and China Postdoctoral Science Foundation. The research reported here was supported by the Natural Science Foundation Project of Chongqing CSTC (Grant no. cstc2018jcyjAX0810) and the Foundation of CQUE (18GZKP02, KY201702A). It was partially supported by Research Foundation of Key Laboratory of Machine Perception and Children's Intelligence Development funded by CQUE (16xjpt07), China.

\section{Competing interests}

The authors declare that there is no conflict of interests regarding the publication of this paper.

\section{Authors' contributions}

All authors read and approved the final manuscript.

\section{Author details}

${ }^{1}$ National and Local Joint Engineering Laboratory of Intelligent Transmission and Control Technology (Chongqing), College of Electronic and Information Engineering, Southwest University, Chongqing, P.R. China. ${ }^{2}$ Department of Computer Science and IT, Ghazi University, D.G. Khan, Pakistan.

\section{Publisher's Note}

Springer Nature remains neutral with regard to jurisdictional claims in published maps and institutional affiliations. 


\section{References}

1. Shah, K., Ali, A., Bushnaq, S.: Hyers-Ulam stability analysis to implicit Cauchy problem of fractional differential equations with impulsive conditions. Math. Methods Appl. Sci. 41(17), 8329-8343 (2018)

2. Wang, J.R., Shah, K., Ali, A.: Existence and Hyers-Ulam stability of fractional nonlinear impulsive switched coupled evolution equations. Math. Methods Appl. Sci. 41(6), 2392-2402 (2018)

3. Li, X., Cao, J.: An impulsive delay inequality involving unbounded time-varying delay and applications. IEEE Trans. Autom. Control 62(7), 3618-3625 (2017)

4. Li, X., Wu, J.: Stability of nonlinear differential systems with state-dependent delayed impulses. Automatica 64, 63-69 (2016)

5. Li, X., Song, S.: Stabilization of delay systems: delay-dependent impulsive control. IEEE Trans. Autom. Control 62(1) 406-411 (2016)

6. Tao, J., Wu, Z.G., Su, H., Wu, Y., Zhang, D.: Asynchronous and resilient filtering for Markovian jump neural networks subject to extended dissipativity. IEEE Trans. Cybern. PP(99), 1-10 (2018)

7. Ying, S., Wu, Z.G., Peng, S., Su, H., Huang, T.: Asynchronous filtering for Markov jump neural networks with quantized outputs. IEEE Trans. Syst. Man Cybern. Syst. PP(99), 1-11 (2018)

8. Yang, X., Peng, D., LV, X., Li, X.: Recent progress in impulsive control systems. Math. Comput. Simul. 155, 244-268 (2019)

9. Lakshmikantham, V., Bainov, D.D., Simeonov, P.S.: Theory of Impulsive Differential Equations. World Scientific, Singapore (1989)

10. Bainov, D.D., Simeonov, P.S.: Systems with Impulse Effect: Stability, Theory and Applications. Wiley, New York (1989)

11. Hirstova, S.G., Bainov, D.D.: Existence of periodic solutions of nonlinear systems of differential equations with impulsive effect. J. Math. Anal. Appl. 125(1), 192-202 (1987)

12. Yang, T.: Impulsive Control Theory (2001)

13. Fan, Z., Gang, L.: Existence results for semilinear differential equations with nonlocal and impulsive conditions. J. Funct. Anal. 258(5), 1709-1727 (2010)

14. Chellaboina, V., Bhat, S.P., Haddad, W.M.: An invariance principle for nonlinear hybrid and impulsive dynamical systems. American Control Conference (2000)

15. Wen, S., Xiao, S., Yan, Z., Zeng, Z., Huang, T.: Adjusting learning rate of memristor-based multilayer neural networks via fuzzy method. IEEE Trans. Comput.-Aided Des. Integr. Circuits Syst. (2018). https://doi.org/10.1109/TCAD.2018.2834436

16. Wen, S., Xiao, S.X., Yang, Y., Yan, Z., Zeng, Z., Huang, T.: Generating realistic videos from keyframes with concatenated gans. IEEE Trans. Circuits Syst. Video Technol. (2018). https://doi.org/10.1109/TSMC.2018.2825021

17. Wen, S., Hu, R., Yang, Y., Huang, T., Zeng, Z., Song, Y.D.: Memristor-based echo state network with online least mean square. IEEE Transactions on Systems, Man, and Cybernetics: Systems (2018) https://doi.org/10.1109/TSMC.2018.2825021

18. Tang, S., Cheke, R.A.: State-dependent impulsive models of integrated pest management (ipm) strategies and their dynamic consequences. J. Math. Biol. 50(3), 257-292 (2005)

19. Zeng, G., Chen, L., Sun, L.: Existence of periodic solution of order one of planar impulsive autonomous system J. Comput. Appl. Math. 186(2), 466-481 (2006)

20. Nie, L., Teng, Z., Torres, A.: Dynamic analysis of an SIR epidemic model with state dependent pulse vaccination. Nonlinear Anal., Real World Appl. 13(4), 1621-1629 (2012)

21. Nersesov, S.G., Chellaboina, V.S., Haddad, W.M.: A generalization of Poincare's theorem to hybrid and impulsive dynamical systems. American Control Conference, 1240-1245 (2002)

22. Ciesielski, K.: On stability in impulsive dynamical systems. Bull. Pol. Acad. Sci., Math. 52(84), 81-91 (2010)

23. Brudnyi, A.: Well-posedness of reset control systems on state-dependent impulsive dynamical systems. Abstr. Appl. Anal. 2012(6), 357-378 (2014)

24. Akhmet, M.: Principles of Discontinuous Dynamical Systems. Springer, New York (2010)

25. Aubin, J.P.: Viability Theory (Systems and Control) (2006)

26. Cardinali, S.: Periodic solutions of nonlinear impulsive differential inclusions with constraints. Proc. Am. Math. Soc. 132(8), 2339-2349 (2004)

27. Cardinali, T., Servadei, R.: On the existence of solutions for nonlinear impulsive periodic viable problems. Open Math. 2(4), 573-583 (2004)

28. Gabor, G.: The existence of viable trajectories in state-dependent impulsive systems. Nonlinear Anal. 72(9), 3828-3836 (2010)

29. Gabor, G.: Viable periodic solutions in state-dependent impulsive problems. Collect. Math. 66(3), 351-365 (2015)

30. Kryszewski, W., Plaskacz, S.: Periodic solutions to impulsive differential inclusions with constraints. Nonlinear Anal. 65(9), 1794-1804 (2006)

31. Akhmet, M.: Discontinuous dynamics with grazing points. Commun. Nonlinear Sci. Numer. Simul. 38, $218-242$ (2016)

32. Bonotto, E.M., Federson, M.: Poisson stability for impulsive semidynamical systems. Nonlinear Anal., Theory Methods Appl. 71(12), 6148-6156 (2009)

33. Haddad, W.M., Chellaboina, V.S.: Nonlinear Dynamical Systems and Control: A Lyapunov-Based Approach. Princeton University Press, Princeton (2011) 